\title{
Smarandache Ruled Surfaces according to Frenet-Serret Frame of a Regular Curve in $E^{3}$
}

\author{
Soukaina Ouarab \\ Department of Mathematics and Computer Science, Hassan II University of Casablanca, Ben M'sik Faculty of Sciences, Morocco
}

Correspondence should be addressed to Soukaina Ouarab; soukaina.ouarab.sma@gmail.com

Received 30 January 2021; Accepted 16 March 2021; Published 1 April 2021

Academic Editor: Chun Gang Zhu

Copyright (C) 2021 Soukaina Ouarab. This is an open access article distributed under the Creative Commons Attribution License, which permits unrestricted use, distribution, and reproduction in any medium, provided the original work is properly cited.

\begin{abstract}
In this paper, we introduce original definitions of Smarandache ruled surfaces according to Frenet-Serret frame of a curve in $E^{3}$. It concerns TN-Smarandache ruled surface, TB-Smarandache ruled surface, and NB-Smarandache ruled surface. We investigate theorems that give necessary and sufficient conditions for those special ruled surfaces to be developable and minimal. Furthermore, we present examples with illustrations.
\end{abstract}

\section{Introduction}

In differential geometry of curves and surfaces [1-3], a ruled surface defines the set of a family of straight lines depending on a parameter. The straight lines mentioned are the rulings of the ruled surface. The general parametric representation of a ruled surface is $\Psi(s, v)=c(s)+v \vec{X}(s)$ where $c(s)$ is a curve through which all rulings pass; it is called the base curve of the surface; the vector $\vec{X}(s)$ defines the ruling direction.

It is well known that ruled surfaces are of great interest to many applications and have contributed in several areas, such as mathematical physics, kinematics, and Computer Aided Geometric Design (CAGD).

Ruled surfaces have been studied differently by an important number of researchers. In [4], the authors constructed the ruled surface whose rulings are constant linear combinations of alternative moving frame vectors of its base curve; they studied the ruled surface properties, characterize it, and presented examples with illustrations in the case of general helices [5] and slant helices [6] as base curves. In [7], the authors constructed the ruled surface whose rulings are constant linear combinations of Darboux frame vectors of a regular curve lying on a regular surface of reference in $E^{3}$. Their point of interest was to make a comparative study between the two surfaces (the regular surface of reference and the new constructed ruled surface) along their common curve.
Also, they investigated properties of the constructed ruled surface. Moreover, they gave examples with illustrations.

The notions of developability and minimalist are two of the most important properties of surfaces.

The ruled surfaces with vanishing Gaussian curvature, which can be transformed into the plane without any deformation and distortion, are called developable surfaces; they form a relatively small subset that contains cylinders, cones, and the tangent surfaces [8-10].

A minimal surface is a surface that locally minimizes its area. It is referred to the fixed boundary curve of a surface area that is minimal with respect to other surfaces with the same boundary. This is equivalent to having zero mean curvature [11-13].

In curve theory, Smarandache curves are one of the special innovated curves that were introduced at the first time in Minkowski space-time by authors in [14]. It is about curves whose position vectors are composed by FrenetSerret frame vectors on another regular curve. In [15-17], we can find several research works about Smarandache curves according to different frames such as the FrenetSerret frame, Bishop frame, and Darboux frame in Euclidean and Minkowski spaces.

The motivation of this work is inspired by ruled surface and Smarandache curve. We are eager to introduce new definitions that combine those two important notions and study their properties. We are also opening up opportunities to 
perceive future works that are relative to applications in differential geometry, physics, and medical science.

In this paper, we are interested in ruled surfaces generated by Smarandache curves according to the Frenet-Serret frame. Indeed, we construct and introduce original definitions of three special ruled surfaces generated by TN-Smarandache curve, TB-Smarandache curve, and NB-Smarandache curve according to the Frenet-Serret frame of an arbitrary regular curve in $E^{3}$. We investigate theorems that give us necessary and sufficient conditions for those three ruled surfaces to be developable and minimal. Finally, we give examples with illustrations.

\section{Preliminaries}

In the Euclidean 3 -space $E^{3}$, we consider the usual metric given by

$$
\langle,\rangle=d x_{1}+d x_{2}+d x_{3},
$$

where $\left(x_{1}, x_{2}, x_{3}\right)$ is a rectangular coordinate system of $E^{3}$.

A curve $c: s \in I \subset \mathbb{R} \rightarrow E^{3}$ is said to be of unit speed (or parameterized by the arc length) if $\left\|c^{\prime}(s)\right\|=\sqrt{\left\langle c^{\prime}(s), c^{\prime}(s)\right\rangle}$ $=1$ for any $s \in I$. For such curves, there is a frame $\{\vec{T}, \vec{N}, \vec{B}\}$ which is called the Frenet-Serret frame, where $\vec{T}=c^{\prime}, \vec{N}=c^{\prime \prime}$ $/\left\|c^{\prime \prime}\right\|$, and $\vec{B}=\vec{T} \times \vec{N}$ are the unit tangent, the principal normal, and the binormal vector of the curve, respectively. The Frenet-Serret formulae of $c(s)$ is given by

$$
\left[\begin{array}{c}
\overrightarrow{T^{\prime}} \\
\overrightarrow{N^{\prime}} \\
\overrightarrow{B^{\prime}}
\end{array}\right]=\left[\begin{array}{ccc}
0 & \kappa & 0 \\
-\kappa & 0 & \tau \\
0 & -\tau & 0
\end{array}\right]\left[\begin{array}{c}
\vec{T} \\
\vec{N} \\
\vec{B}
\end{array}\right]
$$

where $\kappa=\kappa(s)$ are the curvature and the torsion functions of $c(s)$ and they are given by $\kappa=\left\|c^{\prime \prime}\right\|$ and $\tau=\operatorname{det}\left(c^{\prime}, c^{\prime \prime}, c^{\prime \prime \prime}\right) /$ $\left\|c^{\prime \prime}\right\|^{2}$, respectively [2].

Definition 1 (see [18]). TN-Smarandache curves according to the Frenet-Serret frame of the curve $c=c(s)$ are given by

$$
\beta\left(s^{*}(s)\right)=\frac{1}{\sqrt{2}}(\vec{T}(s)+\vec{N}(s)) .
$$

Definition 2 (see [18]). TB-Smarandache curves according to the Frenet-Serret frame of the curve $c=c(s)$ are given by

$$
\gamma\left(s^{*}(s)\right)=\frac{1}{\sqrt{2}}(\vec{T}(s)+\vec{B}(s)) .
$$

Definition 3 (see [18]). NB-Smarandache curves according to the Frenet-Serret frame of the curve $c=c(s)$ are given by

$$
\delta\left(s^{*}(s)\right)=\frac{1}{\sqrt{2}}(\vec{N}(s)+\vec{B}(s))
$$

Let $\Psi:(s, v) \mapsto c(s)+v \vec{X}(s)$ be a ruled surface in $E^{3}$.

Let is denote by $\vec{n}=\vec{n}(s, v)$ the unit normal on ruled surface $\Psi$ at a regular point $\Psi(s, v)$; we have

$$
\vec{n}=\frac{\Psi_{s} \wedge \Psi_{v}}{\left\|\Psi_{s} \wedge \Psi_{v}\right\|}=\frac{\left(c^{\prime}+\overrightarrow{v X^{\prime}}\right) \times \vec{X}}{\left\|\left(c^{\prime}+\overrightarrow{X^{\prime}}\right) \times \vec{X}\right\|},
$$

where $\Psi_{s}=(\partial \Psi(s, v)) / \partial s$ and $\Psi_{v}=(\partial \Psi(s, v)) / \partial v$.

Definition 4 (see [19]). The distribution parameter $\lambda=\lambda(s)$ of ruled surface $\Psi$ is given by

$$
\lambda=\frac{\operatorname{det}\left(c^{\prime}, \vec{X}, \overrightarrow{X^{\prime}}\right)}{\left\|\overrightarrow{X^{\prime}}\right\|^{2}} .
$$

Definition 5 (see [19]). A ruled surface is developable if its distribution parameter vanishes.

The first $I$ and the second $I I$ fundamental forms of ruled surface $\Psi$ at a regular point $\Psi(s, v)$ are defined, respectively, by

$$
\begin{gathered}
I\left(\Psi_{s} d s+\Psi_{v} d v\right)=E d s^{2}+2 F d s d v+G d v^{2} \\
I I\left(\Psi_{s} d s+\Psi_{v} d v\right)=e d s^{2}+2 f d s d v+g d v^{2}
\end{gathered}
$$

where $E=\left\|\Psi_{s}\right\|^{2}, F=\left\langle\Psi_{s}, \Psi_{v}\right\rangle, G=\left\|\Psi_{v}\right\|^{2}, e=\left\langle\Psi_{s s}, \vec{n}\right\rangle, f=$ $\left\langle\Psi_{s v}, \vec{n}\right\rangle$, and $g=\left\langle\Psi_{v v}, \vec{n}\right\rangle=0$.

Definition 6. The Gaussian curvature $K$ and the mean curvature $H$ of ruled surface $\Psi$ at a regular point $\Psi(s, v)$ are given, respectively, by

$$
\begin{aligned}
& K=-\frac{f^{2}}{E G-F^{2}}, \\
& H=\frac{G e-2 F f}{2\left(E G-F^{2}\right)} .
\end{aligned}
$$

Proposition 7 (see [19]). A ruled surface is developable if and only if its Gaussian curvature vanishes.

Proposition 8 (see [19]). A regular surface is minimal if and only if its mean curvature vanishes.

\section{Smarandache Ruled Surfaces according to Frenet-Serret Frame of a Regular Curve in $E^{3}$}

In a first step, we start our section by giving the following new definitions of Smarandache ruled surfaces according to the Frenet-Serret frame of a curve in $E^{3}$.

Definition 9. Let $c=c(s)$ be the $C^{2}$ class differentiable unit speed curve whose Frenet-Serret apparatus is $\{\vec{T}(s), \vec{N}(s), \vec{B}$ 
$(s), \kappa(s), \tau(s)\}$ in $E^{3}$. The ruled surfaces generated by Smarandache curves according to Frenet-Serret of $c=c(s)$ are as follows:

$$
\begin{aligned}
& { }^{1} \Psi(s, v)=\frac{1}{\sqrt{2}}(\vec{T}(s)+\vec{N}(s))+v \vec{B}(s), \\
& { }^{2} \Psi(s, v)=\frac{1}{\sqrt{2}}(\vec{T}(s)+\vec{B}(s))+v \vec{N}(s), \\
& { }^{3} \Psi(s, v)=\frac{1}{\sqrt{2}}(\vec{N}(s)+\vec{B}(s))+v \vec{T}(s) .
\end{aligned}
$$

These ruled surfaces are called TN-Smarandache ruled surface, TB-Smarandache ruled surface, and NB-Smarandache ruled surface, according to the Frenet-Serret frame of the curve $c=c(s)$, respectively.

Following of this section, we investigate theorems that give necessary and sufficient conditions for Smarandache ruled surfaces (Equation (10)) to be developable and minimal. Also, we present an example with illustration for each Smarandache ruled surface.

Theorem 10. The TN-Smarandache ruled surface of Equation (10) is developable if and only if $c=c(s)$ is a plane curve.

Theorem 11. The TN-Smarandache ruled surface of Equation (10) is minimal if and only if the curvature and the torsion of the curve $c=c(s)$ satisfy the equation $\kappa\left(\tau^{2}-\kappa^{\prime}-2 \kappa^{2}\right)+v \sqrt{2}$ $\left(\kappa^{\prime} \tau+2 \kappa^{2} \tau\right)-v^{2} 2 \kappa \tau^{2}=0$.

Proof. Differentiating the first line of Equation (10) with respect to $s$ and $v$, respectively, we get

$$
\begin{aligned}
& { }^{1} \Psi_{s}=-\frac{\kappa}{\sqrt{2}} \vec{T}+\left(\frac{\kappa}{\sqrt{2}}-v \tau\right) \vec{N}+\frac{\tau}{\sqrt{2}} \vec{B}, \\
& { }^{1} \Psi_{v}=\vec{B} .
\end{aligned}
$$

The crossproduct of these two vectors gives the normal vector on the TN-Smarandache ruled surface of Equation (10):

$$
{ }^{1} \Psi_{s} \times{ }^{1} \Psi_{v}=\left(\frac{\kappa}{\sqrt{2}}-v \tau\right) \vec{T}+\frac{\kappa}{\sqrt{2}} \vec{N}
$$

So under regularity condition, the unit normal takes the form

$$
\frac{{ }^{1} \Psi_{s} \times{ }^{1} \Psi_{v}}{\left\|{ }^{1} \Psi_{s} \times{ }^{1} \Psi_{v}\right\|}=\frac{1}{\sqrt{\kappa^{2}-\sqrt{2} \kappa \tau v+\tau^{2} v^{2}}}\left[\left(\frac{\kappa}{\sqrt{2}}-v \tau\right) \vec{T}+\frac{\kappa}{\sqrt{2}} \vec{N}\right] .
$$

Making the norms for Equation (13), we get the components of the first fundamental form of the TN-
Smarandache ruled surface of Equation (10), at regular points, as follows:

$$
\begin{aligned}
& { }^{1} E=\frac{2 \kappa^{2}+\tau^{2}}{2}-\sqrt{2} \kappa \tau \nu+\tau^{2} v^{2}, \\
& { }^{1} F=\frac{\tau}{\sqrt{2}}, \\
& { }^{1} G=1 .
\end{aligned}
$$

Differentiating Equation (13) with respect to $s$ and $v$, respectively, we get

$$
\begin{aligned}
&{ }^{1} \Psi_{s s}=-\left[\frac{\kappa^{\prime}}{\sqrt{2}}+\kappa\left(\frac{\kappa}{\sqrt{2}}-v \tau\right)\right] \vec{T}+\left[\frac{-\kappa^{2}-\tau^{2}+k^{\prime}}{\left.\sqrt{2}-v \tau^{\prime}\right] \vec{N}}\right. \\
&+\left[\frac{\tau^{\prime}}{\sqrt{2}}+\tau\left(\frac{\kappa}{\sqrt{2}}-v \tau\right)\right] \vec{B}, \\
&{ }^{1} \Psi_{v s}=-\tau N, \\
&{ }^{1} \Psi_{v v}=0 .
\end{aligned}
$$

Hence, from Equations (16) and (20), we get the components of the second fundamental form of the TN-Smarandache ruled surface of Equation (10), at regular points, as follows:

$$
{ }^{1} e=-\frac{((\kappa / \sqrt{2})-v \tau)\left(\left(\left(\kappa^{\prime}+\kappa^{2}\right) / \sqrt{2}\right)-v \kappa \tau\right)+\left(\kappa\left(\kappa^{2}+\tau^{2}\right)\right) / 2}{\sqrt{\kappa^{2}-\sqrt{2} \kappa \tau v+\tau^{2} v^{2}}},
$$

${ }^{1} f=-\frac{\kappa \tau}{\sqrt{2} \sqrt{\kappa^{2}-\sqrt{2} \kappa \tau v+\tau^{2} v^{2}}}$,

${ }^{1} g=0$

From Equations (17) and (23), we get the Gaussian curvature and the mean curvature of the TN-Smarandache ruled surface of Equation (10), at regular points, as follows:

$$
\begin{aligned}
& { }^{1} K=-\left[\frac{\kappa \tau}{\sqrt{2}\left(\kappa^{2}-\sqrt{2} \kappa \tau \nu+\tau^{2} v^{2}\right)}\right]^{2}, \\
& { }^{1} H=\frac{-\kappa \kappa^{\prime}-2 \kappa^{3}+\kappa \tau^{2}+v \sqrt{2}\left(\kappa^{\prime} \tau+2 \kappa^{2} \tau\right)-v^{2} 2 \kappa \tau^{2}}{4\left(\kappa^{2}-\sqrt{2} \kappa \tau v+\tau^{2} v^{2}\right)^{3 / 2}},
\end{aligned}
$$

which replies to both the above theorems. 


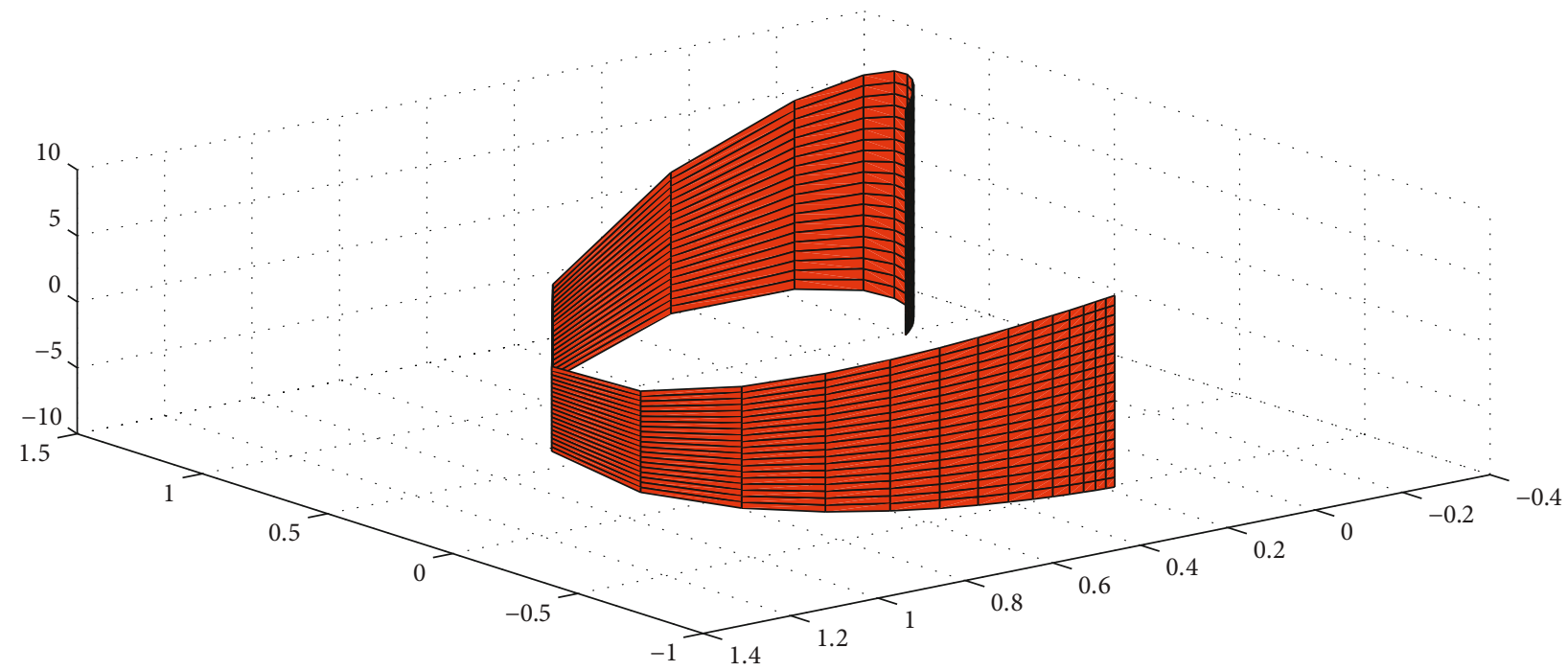

Figure 1: The TN-Smarandache ruled surface (Equation (27)).

Example 12. Let us consider the regular plane curve ${ }^{1} c(s)=$ $\left(3 s-s^{3}, 3 s^{2}, 0\right)$. The TN-Smarandache ruled surface according to the Frenet-Serret frame of the curve ${ }^{1} c(s)$ is given by

$$
\begin{aligned}
{ }^{1} \Psi(s, v)=\frac{1}{\sqrt{2}}\left(\begin{array}{c}
\frac{1-s^{2}}{\sqrt{\left(1-s^{2}\right)^{2}+4 s^{2}}}-\frac{s}{\sqrt{1+s^{2}}} \\
\frac{2 s}{\sqrt{\left(1-s^{2}\right)^{2}+4 s^{2}}}-\frac{1}{\sqrt{1+s^{2}}} \\
0 \\
0
\end{array}\right) \\
+v\left(\begin{array}{c}
1-s+2 s^{2} \\
\frac{1}{\sqrt{\left[\left(1-s^{2}\right)^{2}+4 s^{2}\right]\left[\sqrt{1+s^{2}}\right]}}
\end{array}\right) .
\end{aligned}
$$

It is developable but not minimal at its regular points. Figure 1 is the illustration of Equation (27) drawn for $(s, v)$ $\epsilon]-2 \pi, 2 \pi[\times]-4,4[$.

Theorem 13. The TB-Smarandache ruled surface of Equation (10) is developable.

Theorem 14. The TB-Smarandache ruled surface of Equation (10) is minimal if and only if the curve $c=c(s)$ is a general helix.

Proof. Differentiating the second line of Equation (10) with respect to $s$ and $v$, respectively, we get

$$
\begin{aligned}
& { }^{2} \Psi_{s}=-v \kappa \vec{T}+\frac{\kappa-\tau}{\sqrt{2}} \vec{N}+v \tau \vec{B}, \\
& { }^{2} \Psi_{v}=\vec{N}
\end{aligned}
$$

which implies that the normal vector on the TB-Smarandache ruled surface of Equation (10) is

$$
{ }^{2} \Psi_{s} \times{ }^{2} \Psi_{v}=-v \tau \vec{T}-v \kappa
$$

So under regularity condition, the unit normal is given by

$$
\frac{{ }^{2} \Psi_{s} \times{ }^{2} \Psi_{v}}{\left\|^{2} \Psi_{s} \times{ }^{2} \Psi_{v}\right\|}=\mp \frac{\tau \vec{T}+\kappa \vec{B}}{\sqrt{\left(\kappa^{2}+\tau^{2}\right)}} .
$$

Making the norms for Equation (28), we get the components of the first fundamental form of the TB-Smarandache ruled surface of Equation (10), at regular points:

$$
\begin{aligned}
& { }^{2} E=\frac{(\kappa-\tau)^{2}}{2}+v^{2}\left(\kappa^{2}-\tau^{2}\right), \\
& { }^{2} F=\frac{\kappa-\tau}{\sqrt{2}}, \\
& { }^{2} G=1 .
\end{aligned}
$$

Differentiating Equation (28) with respect to $s$ and $v$, respectively, we get

$$
\begin{aligned}
& { }^{2} \Psi_{s s}=-\left[\frac{\kappa(\kappa-\tau)}{\sqrt{2}}+v \kappa^{\prime}\right] \vec{T}+\left[\frac{\left(\kappa^{\prime}+\tau^{\prime}\right)}{\sqrt{2}}-v\left(\kappa^{2}-\tau^{2}\right)\right] \vec{N} \\
& +\left[\frac{\tau(\kappa-\tau)}{\sqrt{2}}+v \tau^{\prime}\right] \vec{B}, \\
& { }^{2} \Psi_{v s}=-\kappa \vec{T}+\tau \vec{B} \\
& { }^{2} \Psi_{v v}=0 .
\end{aligned}
$$




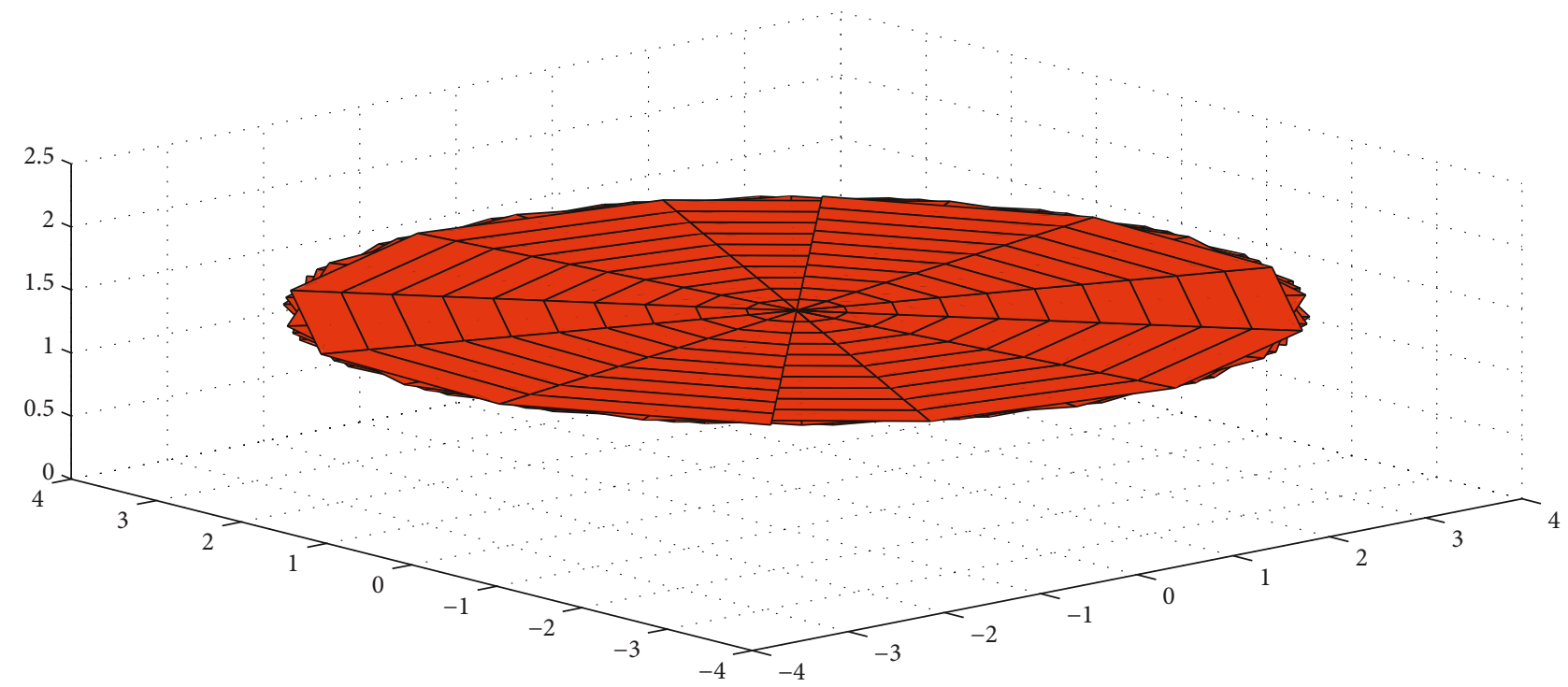

Figure 2: The TB-Smarandache ruled surface (Equation (42)).

Hence, from Equations (31) and (35), we get the components of the second fundamental form of the TBSmarandache ruled surface of Equation (10) at regular points:

$$
\begin{aligned}
& { }^{2} e=\mp v \frac{\kappa^{2}\left(\begin{array}{l}
\tau \\
\kappa
\end{array}\right),}{\sqrt{\kappa^{2}+\tau^{2}}}, \\
& { }^{2} f=0, \\
& { }^{2} g=0 .
\end{aligned}
$$

From Equations (32) and (38), we get the Gaussian curvature and the mean curvature of the TB-Smarandache ruled surface of Equation (10), at regular points, as follows:

$$
\begin{aligned}
& { }^{2} K=0, \\
& { }^{2} H=\mp \frac{\kappa^{2}\left(\begin{array}{l}
\tau \\
\kappa
\end{array}\right)}{2 v\left(\kappa^{2}+\tau^{2}\right)^{3 / 2}},
\end{aligned}
$$

which replies to both the above theorems.

Corollary 15. If $c(s)$ is a general helix, the TB-Smarandache ruled surface of Equation (10) is developable and minimal.

Example 16. Here, we chose the regular curve ${ }^{2} c(s)=1 /$ $\sqrt{2}(\sin (\sqrt{2 s}) / \sqrt{2},-\cos (\sqrt{2 s}) / \sqrt{2}, s)$ as a general helix.
Then, the TB-Smarandache ruled surface according to the Frenet-Serret frame of ${ }^{2} c(s)$ is

$$
{ }^{2} \Psi(s, v)=\frac{1}{2}\left(\begin{array}{c}
\cos (\sqrt{2 s})-\sin (\sqrt{2 s}) \\
\cos (\sqrt{2 s})+\sin (\sqrt{2 s}) \\
1
\end{array}\right)+v\left(\begin{array}{c}
-\cos (\sqrt{2 s}) \\
-\sin (\sqrt{2 s}) \\
1
\end{array}\right)
$$

It is developable and minimal at its regular points. Figure 2 is the illustration of Equation (42) drawn for $(s, v)$ $\epsilon]-2 \pi, 2 \pi[\times]-4,4[$.

Theorem 17. The NB-Smarandache ruled surface of Equation (10) is developable if and only if $c=c(s)$ is a plane curve.

Theorem 18. The NB-Smarandache ruled surface of Equation (10) is minimal if and only if the curvature and the torsion of the curve $c=c(s)$ satisfy the equation

$$
\tau \frac{2 \kappa^{2} \tau-2 \tau^{2}-\kappa^{2}}{\sqrt{2}}+v\left(\kappa^{\prime} \tau+2 \kappa \tau^{2}-\kappa^{2} \tau^{\prime}\right)-\sqrt{2} v^{2} \kappa^{2} \tau=0
$$

Proof. Differentiating the third line of Equation (10) with respect to $s$ and $v$, respectively, we get

$$
\begin{aligned}
& { }^{3} \Psi_{s}=-\frac{\kappa}{\sqrt{2}} \vec{T}+\left(-\frac{\tau}{\sqrt{2}}+v \kappa\right) \vec{N}+\frac{\tau}{\sqrt{2}} \vec{B}, \\
& { }^{3} \Psi_{v}=\vec{T} .
\end{aligned}
$$




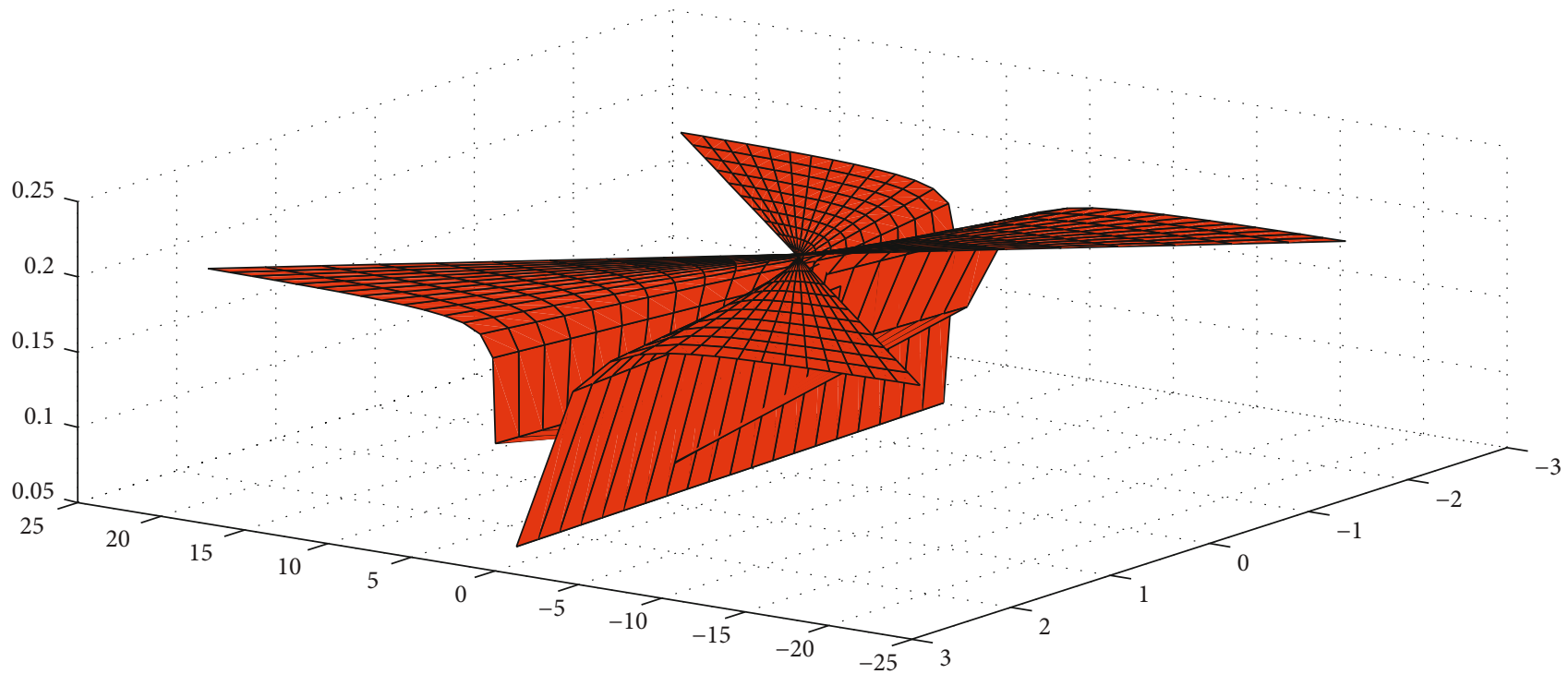

Figure 3: The NB-Smarandache ruled surface (Equation (58)).

Realizing the crossproduct of those two vectors, we get the normal vector on the NB-Smarandache ruled surface of Equation (10):

$$
{ }^{3} \Psi_{s} \times{ }^{3} \Psi_{v}=\frac{\tau}{\sqrt{2}} \vec{N}+\left(\frac{\tau}{\sqrt{2}}-v \kappa\right) \vec{B}
$$

So under regularity condition, the unit normal takes the form

$\frac{{ }^{3} \Psi_{s} \times{ }^{3} \Psi_{v}}{\left\|\Psi_{s} \times{ }^{3} \Psi_{v}\right\|}=\frac{1}{\sqrt{\tau^{2} / 2+((\tau / \sqrt{2})-v \kappa)^{2}}}\left[\frac{\tau}{\sqrt{2}} \vec{N}+\left(\frac{\tau}{\sqrt{2}}-v \kappa\right) \vec{B}\right]$

Making the norms for Equation (44), we get the components of the first fundamental form of the NB-Smarandache ruled surface of Equation (10), at regular points, as follows:

$$
{ }^{3} E=\frac{\kappa^{2}+\tau^{2}}{2}+\left(-\frac{\tau}{\sqrt{2}}+v \kappa\right)^{2}
$$

$$
\begin{aligned}
& { }^{3} F=-\frac{\kappa}{\sqrt{2}}, \\
& { }^{3} G=1 .
\end{aligned}
$$

Differentiating Equation (44) with respect to $s$ and $v$, respectively, we get

$$
\begin{aligned}
{ }^{3} \Psi_{s s}= & -\left[\frac{\kappa^{\prime}-\kappa \tau}{\sqrt{2}}+v \kappa^{2}\right] \vec{T}+\left[\frac{\kappa^{2}+\tau^{2}+\tau^{\prime}}{\sqrt{2}}+v \kappa^{\prime}\right] \vec{N} \\
& +\left[\frac{\tau^{\prime}+\tau^{2}}{\sqrt{2}}+v \kappa \tau\right] \vec{B}, \\
{ }^{3} \Psi_{v s}= & -\kappa \vec{N}, \\
{ }^{3} \Psi_{v v}= & 0 .
\end{aligned}
$$

Hence, from Equations (47) and (51), we get the components of the second fundamental form of the NBSmarandache ruled surface of Equation (10), at regular points, as follows:

$$
\begin{aligned}
& { }^{3} e=\frac{\tau / \sqrt{2}\left[-\left(\left(\kappa^{2}+\tau^{2}+\tau^{\prime}\right) / \sqrt{2}\right)+v \kappa^{\prime}\right]+((\tau / \sqrt{2})-v \kappa)\left[\left(\left(\tau^{\prime}+\tau^{2}\right) / \sqrt{2}\right)+v \kappa \tau\right]}{\sqrt{\tau^{2} / 2+((\tau / \sqrt{2})-v \kappa)^{2}}}, \\
& { }^{3} f=\frac{\kappa \tau}{\sqrt{\tau^{2}+(\tau-\sqrt{2} v \kappa)^{2}}}, \\
& { }^{3} g=0 .
\end{aligned}
$$


From Equations (48) and (54), we get the Gaussian curvature and the mean curvature of the NB-Smarandache ruled surface of Equation (10), at regular points, as follows:

$$
\begin{aligned}
& { }^{3} K=-\left[\frac{\sqrt{2} \kappa \tau}{\tau^{2}+(\tau-\sqrt{2} v \kappa)^{2}}\right]^{2}, \\
& { }^{3} H=2 \frac{\left[\tau\left(2 \kappa^{2} \tau-2 \tau^{2}-\kappa^{2}\right) / \sqrt{2}\right]+\left[v\left(\kappa^{\prime} \tau+2 \kappa \tau^{2}-\kappa^{2} \tau^{\prime}-\sqrt{2} v^{2} \kappa^{2} \tau\right)\right]}{\left[\tau^{2}+(\tau-\sqrt{2} v \kappa)^{2}\right]^{3 / 2}},
\end{aligned}
$$

which replies to both the above theorems.

Corollary 19. If the NB-Smarandache ruled surface of Equation (10) is developable, it is minimal.

Example 20. Let us consider the plane curve ${ }^{3} c(s)=\left(s^{2}, s^{3}, 0\right)$. Thus, the NB-Smarandache ruled surface according to the Frenet-Serret frame of ${ }^{3} c(s)$ is parametrized by

$$
\Psi(s, v)=\frac{1}{\sqrt{2\left(4+36 s^{2}\right)}}\left(\begin{array}{c}
2 \\
6 s \\
\frac{6 s^{2}}{\sqrt{4 s^{2}+9 s^{4}}}
\end{array}\right)+\frac{v}{\sqrt{4 s^{2}+9 s^{4}}}\left(\begin{array}{c}
2 s \\
3 s^{2} \\
0
\end{array}\right) .
$$

It is developable and minimal. Figure 3 is the illustration of Equation (58) which is drawn for $(s, v) \in]-2 \pi, 2 \pi[\times]-4,4[$.

\section{Conclusion}

The main results of the present work assure the following:

(i) The TN-Smarandache ruled surface according to the Frenet-Serret frame is developable (resp., minimal) if $c(s)$ is a plane curve (resp., $\kappa$ and $\tau$, satisfy special equation)

(ii) The NB-Smarandache ruled surface according to the Frenet-Serret frame is developable (resp., minimal) if $c(s)$ is a plane curve (resp., $\kappa$ and $\tau$, satisfy special equation)

(iii) The investigated theorems prove that developability and minimality conditions of the introduced Smarandache ruled surfaces according to the Frenet-Serret frame are related to differential properties of the reference curve. Therefore, we can easily constate that to construct a developable Smarandache ruled surface or a minimal Smarandache ruled surface according to the Frenet-Serret frame, we just need to make the right choice for the reference curve $c=c(s)$

\section{Data Availability}

No data were used to support the study.

\section{Conflicts of Interest}

The author declares that there are no conflicts of interest.

\section{References}

[1] D. J. Struik, Lectures on Classical Differential Geometry, Dover, AddisonWesley, 2nd edition, 1988.

[2] M. P. Do Carmo, Differential Geometry of Curves and Surfaces, Prentice Hall, Englewood Cliffs, NJ, 1976.

[3] S. Yilmaz and M. Turgut, "On the differential geometry of the curves in Minkowski spacetime," International Journal of Contemporary Mathematical Sciences, vol. 27, no. 3, pp. 13431349, 2008.

[4] S. Ouarab, A. O. Chahdi, and M. Izid, "Ruled surfaces with alternative moving frame in Euclidean 3-space," International Journal of Mathematical Sciences and Engineering Applications, vol. 12, no. 2, pp. 43-58, 2018.

[5] A. T. Ali, "Position vectors of general helices in Euclidean 3space," Bulletin of Mathematical Analysis and Applications, vol. 3, no. 2, pp. 198-205, 2011.

[6] A. T. Ali, "Position vectors of slant helice in Euclidean space $E^{3}$," Journal of the Egyptian Mathematical Society, vol. 20, no. 1, pp. 1-6, 2012.

[7] S. Ouarab, A. O. Chahdi, and M. Izid, "Ruled surface generated by a curve lying on a regular surface and its characterizations," Journal for Geometry and Graphics, vol. 24, no. 2, pp. 257-267, 2020.

[8] G. Hu, H. Cao, J. Wu, and G. Wei, "Construction of developable surfaces using generalized C-Bézier bases with shape parameters," Computational and Applied Mathematics, vol. 39, no. 3, 2020.

[9] T. G. Nelson, T. K. Zimmerman, S. P. Magleby, R. J. Lang, and L. L. Howell, "Developable mechanisms on developable surfaces," Science Robotics, vol. 4, no. 27, article eaau5171, 2019.

[10] P. Alegre, K. Arslan, A. Carriazo, C. E. Murathan, and G. Öztürk, "Some special types of developable ruled surface," Hacettepe Journal of Mathematics and Statistics, vol. 39, no. 3, pp. 319-325, 2010.

[11] W. Y. Lam, "Minimal surfaces from infinitesimal deformations of circle packings," Advances in Mathematics, vol. 362, pp. 106-939, 2020.

[12] T. H. Colding and C. de Lellis, "The min-max construction of minimal surfaces," Surveys in Differential Geometry, vol. 8, no. 1, pp. 75-107, 2003.

[13] J. L. M. Barbosa and A. G. Colares, Minimal Surfaces in $R^{3}$, Springer Verlag, Berlin heidelberg, 1986.

[14] O. Bektas and S. Yuce, "Special Smarandache curves according to Darboux frame in Euclidean 3- space," Romanian Journal of Mathematics and Computer Science, vol. 3, no. 1, pp. 48-59, 2013.

[15] E. M. Solouma and W. M. Mahmoud, "On spacelike equiformBishop Smarandache curves on $S_{1}^{2}$," Journal of the Egyptian Mathematical Society, vol. 27, no. 1, 2019.

[16] M. Cetin, Y. Tuncer, and M. K. Karacan, "Smarandache curves according to Bishop frame in Euclidean 3-space," General Mathematics Notes, vol. 20, no. 2, pp. 50-66, 2014. 
[17] M. Turgut and S. Yilmaz, "Smarandache curves in Minkowski space-time," International Journal of Mathematical Combinatorics, vol. 3, pp. 51-55, 2008.

[18] A. T. Ali, "Special Smarandache curves in the Euclidean space," International Journal of Mathematical Combinatorics, vol. 2, pp. 30-36, 2010.

[19] B. O. Neill, Sem-Riemannian Geometry, Academic press, New York, 1983. 